\title{
Modes d'utilisation des terres et stock de carbone organique du sol dans le bassin supérieur de Magou au Bénin
}

\author{
Christophe Codjo ATCHADA ${ }^{1^{*}}$, Alex Gbêliho ZOFFOUN² ${ }^{2}$, Tobi Moriaque AKPLO ${ }^{3}$, \\ Anastase Hessou AZONTONDE ${ }^{4}$, Agossou Brice TENTE ${ }^{1}$ et Julien Gaudence DJEGO ${ }^{5}$ \\ ${ }^{1}$ Laboratoire de Biogéographie et Expertise Environnementale (LABEE), Département de Géographie et \\ Aménagement du Territoire (DGAT), Université d'Abomey-Calavi ; BP 698 Abomey-Calavi (BENIN) \\ ${ }^{2}$ Institut National des Recherches Agricoles du Bénin (INRAB) à Agonkanmey ; 01BP 884 Cotonou (BENIN) \\ ${ }^{3}$ Laboratoire de Microbiologie des Sols et d'Ecologie Microbienne/ Faculté des Sciences \\ Agronomiques/Université d'Abomey-Calavi ; 01 BP 526 Cotonou (BENIN) \\ ${ }^{4}$ Laboratoire des Sciences du Sol, Eaux et Environnement/Institut National des Recherches Agricoles du Bénin \\ à Agonkanmey ; 01 BP 988 Cotonou (BENIN) \\ ${ }^{5}$ Laboratoire d'Ecologie Appliquée, Faculté des Sciences Agronomiques/Université d'Abomey-Calavi; 01 BP \\ 526 Cotonou (BENIN) \\ *Adresse correspondante ; E-mail: christophe.atchada@gmail.com; Tel: (229) 96003319/95405973 ; \\ BP 1349 Abomey-Calavi (République du Bénin).
}

\section{RESUME}

Avec la dégradation des terres liée à la croissance démographique, les rendements agricoles baissent dans le Nord-Ouest du Bénin. L'étude vise à évaluer l'impact des différents modes d'utilisation des terres sur le stock de carbone organique du sol dans le bassin supérieur de Magou. A partir d'un choix raisonné sur trois sites suivant les modes d'utilisation des terres, la profondeur et la topo-séquence, 108 échantillons de sol ont été prélevés. La teneur en carbone organique du sol a été déterminée par la méthode de Walkley et Black et leur variation évaluée par une analyse de variance (ANOVA) au seuil de 5\%. Les résultats montrent que le plus élevé et le plus faible stock de carbone ont été obtenus respectivement dans les formations naturelles (29,51 t/ha) et les champs $(16,76 \mathrm{t} / \mathrm{ha})$. Le stock de carbone organique du sol a significativement varié suivant la profondeur avec un fort stock de carbone entre $0-10 \mathrm{~cm}$. Concernant la topo-séquence, le stock le plus élevé est en haut de pente et le plus faible est à mi- pente. Par conséquent, la stabilisation du carbone organique du sol s'impose pour la durabilité de l'agriculture et la réduction des gaz à effet de serre.

(C) 2018 International Formulae Group. All rights reserved

Mots clés: rendement, sol dégradé, teneur en carbone, bassin Magou, Bénin.

\section{Land use patterns and soil organic carbon stock in the Magou Upper Basin in Benin}

\section{ABSTRACT}

With land degradation linked to population growth, agricultural yields are falling in northwestern Benin. The study aimed at evaluating the impact of different land use patterns on soil organic carbon stock in the Magou Upper Basin. From a reasoned choice on three sites according to land use patterns, depth and topo-sequence, 108 
soil samples were collected. Soil organic carbon content was determined by the Walkley and Black method and their variation was assessed by analysis of variance at the 5\% threshold. Results showed that the highest and lowest carbon stocks were obtained respectively in the natural formations (29.51 t / ha) and the fields (16.76 t / ha). The soil organic carbon stock has significantly varied depending on the depth with a high carbon stock between $0-10 \mathrm{~cm}$. For the topo-sequence, the highest stock is at the top of the slope and the lowest is at midslope. Hence, Soil organic carbon stabilization is needed for the sustainability of agriculture and the reduction of greenhouse gases.

(C) 2018 International Formulae Group. All rights reserved

Keywords: yield, degraded soil, carbon content, Magou Upper Basin, Benin.

\section{INTRODUCTION}

Le stockage de Carbone Organique du Sol (COS) est un sujet de prime importance dans les négociations internationales visant à lutter contre le changement climatique au travers d'une réduction d'émissions de Gaz à Effet de Serre (GES) (Tosser et al., 2013). En effet, l'évolution à moyen et long termes des stocks organiques des sols est un indicateur à prendre en compte dans le jugement sur la durabilité des agroécosystèmes et la protection de l'environnement. Le stockage potentiel du carbone dans les sols dépend étroitement du mode d'occupation des terres et de leur gestion (Balesdent et al., 2000). Le changement d'utilisation des terres induit également des pertes de carbone dans les sols (Herfurth, 2015). Depuis plusieurs décennies, l'augmentation des besoins alimentaires a entraîné des besoins accrus des populations rurales en terres fertiles à cause de la pression foncière liée à l'explosion démographique que connaissent en général les pays de l'Afrique de l'Ouest (Fermont et al., 2008). Au Bénin, le problème de la baisse de la fertilité des sols est une préoccupation aussi bien pour les agriculteurs qui se heurtent au coût élevé des intrants que pour les chercheurs dont les travaux de recherche visent à maintenir ou à restaurer la fertilité des sols dégradés pour une intensification de la production agricole (Saïdou et al., 2009). Dans le Nord-Ouest du Bénin, le taux de carbone baisse dans le temps passant de 2,44\% dans l'horizon superficiel à $1,90 \%$ voire $0,3 \%$ sur les plateaux (Kombiénou et al., 2014). La partie inférieure du bassin de la rivière Magou étant sous la protection de la Réserve de Biosphère de la Pendjari, le bassin supérieur subit une dégradation dont l'ampleur n'est pas encore bien maîtrisée. L'analyse de l'évolution des données de production de certaines principales cultures dans cette zone sur les deux dernières décennies montre une baisse des rendements agricoles d'en moyenne 10\% (DSA, 2017). De ces constats découlent l'interrogation suivante : comment varient la teneur en COS et le stock de COS selon la profondeur, la topo-séquence, la texture et les modes d'utilisation des terres (MUT) dans le bassin supérieur de Magou (BSM) ? L'objectif de l'étude est d'évaluer l'impact des différents modes d'utilisation des terres sur le stock de carbone organique du sol dans le bassin supérieur de Magou. Cela permettra d'apprécier la place des usages des terres dans la perte de la fertilité organique des sols dans cette zone afin d'apporter les mesures correctives appropriées pour une gestion durable des terres au profit des agriculteurs, des aménagistes et des décideurs.

\section{MATÉRIEL ET MÉTHODES Milieu d'étude}

Le bassin de Magou est le principal sous bassin de la rivière Pendjari dans le Nord-Ouest de la République du Bénin. Sa partie supérieure se situe entre $10^{\circ} 21^{\prime} 10^{\prime \prime}$ et $10^{\circ} 44^{\prime} 50^{\prime \prime}$ de latitude Nord et entre $0^{\circ} 59^{\prime} 38^{\prime \prime}$ ' et $1^{\circ} 16$ ' $57^{\prime \prime}$ ' de longitude Est. Il couvre, à l'exutoire de Tiélé, 82869 ha et s'étend sur les communes de Tanguiéta, de Matéri, de Kobly et de Boukoumbé (Figure 1). Le climat est soudanien avec en moyenne $1000 \mathrm{~mm}$ à 1100 $\mathrm{mm}$ de pluie par an (Nukpo, 2016) et le relief est influencé par la chaîne de l'Atakora. Les sols sont pour la plupart ferrugineux lessivés. La végétation évolue de la forêt claire ou galerie à la savane arbustive. Elle est 
annuellement soumise aux feux de végétation et continuellement assujettie au déboisement et au surpâturage. L'agriculture et l'élevage constituent les activités dominantes.

\section{Technique d'échantillonnage et de prélèvement}

Le bassin supérieur de Magou est choisi en raison de la dégradation de ses terres agricoles en bordure de la Réserve de Biosphère de la Pendjari. Trois sites ont été retenus, en fonction des trois principaux types de sols exploités par les agriculteurs en termes de superficie et de représentativité concernant la diversité des modes d'utilisation des terres, de façon à couvrir géographiquement le BSM (Figure 1). Le nombre d'échantillons collectés par mode d'utilisation a été déterminé par la formule de Dagnelie (1998) et Glèlè Kakaï et al. (2016).

$$
\mathrm{N}=\left[\mathrm{t}_{1-\alpha / 2} \times(\mathrm{CV} / \mathrm{d})\right]^{2}
$$

$\mathrm{CV}=$ coefficient de variation du paramètre. $\mathrm{Le}$ $\mathrm{CV}$ a été déterminé suite à un échantillonnage et analyse préliminaire ; $d=$ marge d'erreur d'estimation du paramètre à estimer; $5 \leq \mathrm{d} \leq$ $15 \%$. Pour cette étude, nous avons utilisé une marge d'erreur de $10 \% ; \mathrm{t}_{1-\alpha / 2}$ est la valeur de la statistique $t$ de la distribution de Student pour un risque alpha de 0,$05 ; \mathrm{t}_{1-\alpha / 2} \approx 2$.

En appliquant cette formule, 36 carottes de sol ont été prélevées sur une profondeur de $30 \mathrm{~cm}$, répétés sur 3 sites dans le bassin versant, soit un total de 108 échantillons (Tableau 1). Les échantillons pris à la tarière hollandaise par mode d'utilisation des terres (formation naturelle, plantation, jachère et champ), par topo-séquence (haut de pente, mi-pente et bas de pente) sont faits suivant trois profondeurs $(0-10 \mathrm{~cm}, 10-20 \mathrm{~cm}$ et $20-30 \mathrm{~cm})$. En dehors des formations naturelles âgées de plus de 7 ans, les autres modes d'utilisation sont âgés de 7 ans en moyenne.

\section{Méthodes d'analyse des échantillons}

Le carbone organique a été déterminé par la méthode de Walkley et Black (1934) modifiée par Tekalign et al. (1991) qui consiste en une digestion du sol avec le dichromate de potassium $(1 \mathrm{~N})$ en milieu acide sulfurique concentré (Kouelo et al., 2015). La méthode d'estimation du carbone utilisée dans le cadre de cette étude correspond à l'approche développée par le GIEC et utilisée par Saïdou et al. (2012) et Bello et al. (2017). La méthode employée pour calculer les stocks de carbone du sol consiste à mesurer la teneur en carbone organique total à différentes profondeurs du sol et à transformer ces données, en prenant en compte la densité apparente et la pierrosité ou charge en éléments grossiers du sol. Dans un premier temps, nous avons déterminé le stock de carbone organique sur chaque profondeur par la formule suivante :

$\operatorname{COS}_{\text {Profondeur }}=([\mathrm{COS}] \times$ DA $\times$ P $\times(1-$ frag $) \times 10)$

Ces valeurs de stock ont été utilisées pour évaluer la variation du stock de COS suivant la profondeur.

Ensuite, nous avons déterminé le stock de carbone organique sur la profondeur 0-30 $\mathrm{cm}$ par la formule suivante :

$\mathrm{COS}=\sum_{\text {Profondeur }=1}^{\text {Prondeu }}\left(\mathrm{COS}_{\text {profondeur }}\right)$

Avec : $\operatorname{COS}=$ stock de carbone organique des sols représentatifs du type de formation végétale et du sol étudié à un point donné (exprimée en tonne C./ha); COSProfondeur $=$ teneur en carbone organique pour une profondeur donnée du sol (tonne C/ha); [COS] $=$ concentration du carbone organique dans une masse de sol donnée, obtenue par analyses au laboratoire $(\mathrm{g} \mathrm{C} \mathrm{kg} / \mathrm{sol}) ; \mathbf{D A}=$ Densité Apparente (tonne de $\mathrm{sol} / \mathrm{m}^{3}$ ) $; \mathbf{P}=$ profondeur de l'horizon ou épaisseur de la couche du sol (m) ; frag = pourcentage de volume de fragments grossiers $/ 100 ; \mathrm{n}=30 \mathrm{~cm}$.

La mesure de la densité apparente a été faite en prélevant une portion de sol non tassé grâce à un cylindre de densité calibrée de volume connu dont on a pesé la masse après dessiccation à $105{ }^{\circ} \mathrm{C}$ à l'étuve. La densité apparente DA a été calculée par le rapport du poids sec par le volume du cylindre. $\mathrm{DA}=\mathrm{p} / \mathrm{V}$ avec $\mathrm{p}$ : poids sec du sol en $\mathrm{g} ; \mathrm{V}$ (volume en $\left.\mathrm{cm}^{3}\right)=3,14 \times \mathrm{h} \times \mathrm{r}^{2} ; \mathrm{h}=$ Profondeur de l'échantillonnage et $r=$ rayon de la base du cylindre.

Les fractions granulométriques du sol (sable, limon et argile) ont été déterminées par la méthode de pipette de Robison (Akplo et al., 2017). Les fractions considérées sont l'argile $(0-2 \mu \mathrm{m})$; limon $(2-50 \mu \mathrm{m})$ et sable $(50-2000$ $\mu \mathrm{m})$. 


\section{Traitement statistique}

Les analyses statistiques ont été effectuées avec le logiciel $\mathrm{R}$ version 3.2.2. Les données collectées et les paramètres calculés ont été soumis à une analyse de variance (ANOVA) pour vérifier d'éventuelles différences statistiques. L'effet des modes d'utilisation des terres et de la topo-séquence ainsi que leur combinaison ont été testés sur le stock de carbone organique calculé sur la profondeur de $0-30 \mathrm{~cm}$ suivant la procédure "'General Linear Model'. L'effet de la profondeur a été testé sur les sections de $10 \mathrm{~cm}$ $(0-10 \mathrm{~cm} ; 10-20 \mathrm{~cm}$ et $20-30 \mathrm{~cm})$. La séparation des moyennes a été faite en utilisant le test de Student-Newman Keuls, basé sur le calcul de la plus petite amplitude significative (PPAS) au seuil de 5\%.

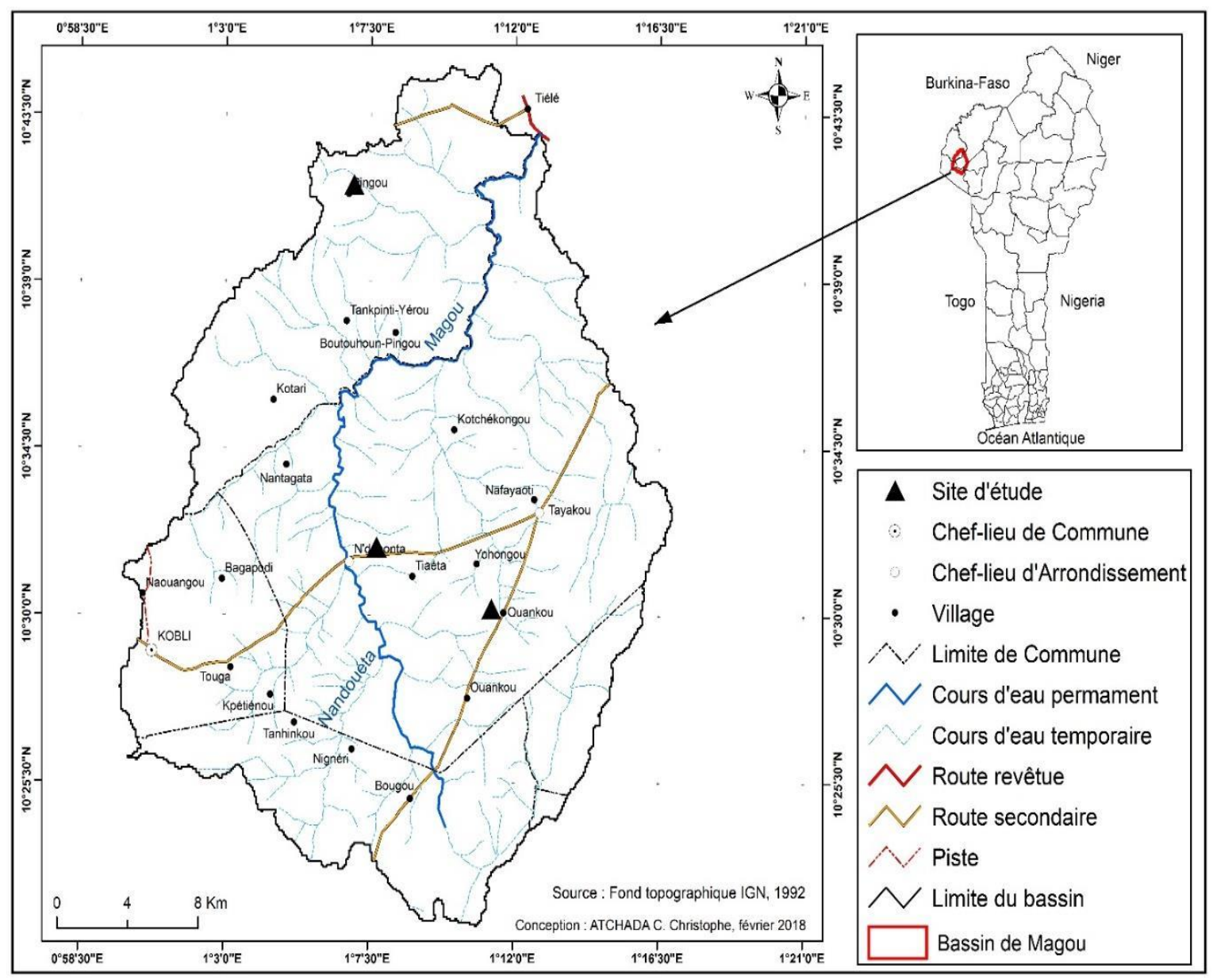

Figure 1: Situation du bassin supérieur de Magou.

Tableau 1 : Détail du nombre d'échantillons prélevés.

\begin{tabular}{lcc}
\hline Modes d'utilisation des terres & Coefficient de variation & Nombre d'échantillons prélevés \\
\hline Champ & 0,15 & 9 \\
\hline Plantation & 0,14 & 9 \\
\hline Formation naturelle & 0,16 & 9 \\
\hline Jachère & 0,15 & 9 \\
\hline Total & & 36 \\
\hline
\end{tabular}




\section{RESULTATS}

\section{Variation du stock de COS en fonction des modes d'utilisation des terres, de la topo- séquence et de la profondeur}

Le Tableau 2 présente les résultats de l'analyse de variance de l'effet des modes d'utilisation des terres, de la topo-séquence et de la profondeur sur le stock de carbone organique du sol. Il ressort de ces résultats que le MUT et la profondeur ont significativement $(\mathrm{p}<0,05)$ influencé le stock de carbone organique du sol (Tableau 2). La comparaison des moyennes montre que le plus fort stock de carbone organique a été obtenu dans les formations naturelles $(29,51 \mathrm{t} / \mathrm{ha})$ et le plus faible stock a été obtenu dans les champs $(16,76 \mathrm{t} / \mathrm{ha})$. Le stock de carbone organique du sol a significativement décru entre la profondeur $0-10 \mathrm{~cm}$ et les autres profondeurs mais reste constant entre la profondeur 10-20 $\mathrm{cm}$ et la profondeur $20-30 \mathrm{~cm}$. Par ailleurs, entre ces deux profondeurs, il y a une différence arithmétique (Tableau 3). Le stock de carbone organique entre les différentes positions de la topo-séquence n'est pas significativement $(\mathrm{p}>0,05)$ différent mais le plus fort stock a été obtenu en haut de pente $(24,63 \mathrm{t} / \mathrm{ha})$ contre $21,83 \mathrm{t} / \mathrm{ha}$ et $18,44 \mathrm{t} / \mathrm{ha}$ respectivement en bas de pente et à mi-pente.

\section{Variation du stock de COS suivant la profondeur et le mode d'utilisation des terres}

La Figure 2 présente l'effet interactif du mode d'utilisation des terres et de la profondeur sur le stock de COS. Pour tous les MUT, c'est la profondeur 0-10 cm qui a donné le stock de COS le plus élevé. Sur cette profondeur $(0-10 \mathrm{~cm})$, le stock de COS des formations naturelles $(16,81$ t/ha $)$ est significativement supérieur à ceux des plantations $(12,39 \mathrm{t} / \mathrm{ha})$, des jachères $(9,31 \mathrm{t} / \mathrm{ha})$ et des champs $(7,99 \mathrm{t} / \mathrm{ha})$. Au niveau de la profondeur $10-20 \mathrm{~cm}$, la différence n'est pas significative entre les différents MUT. Néanmoins, le stock de COS des formations naturelles est 1,42 fois supérieur à celui des autres modes d'utilisation des terres. Pour ces derniers, les stocks de COS obtenus sont de $5,55 \mathrm{t} / \mathrm{ha} ; 5,35 \mathrm{t} / \mathrm{ha}$ et $5,026 \mathrm{t} / \mathrm{ha}$ respectivement pour les plantations, la jachère et les champs. La profondeur $20-30 \mathrm{~cm}$ a eu un effet similaire. Les formations naturelles ont donné un stock de COS significativement supérieur à celui des autres MUT. La différence n'est pas significative entre les champs et les jachères pour cette profondeur.

\section{Variation du stock de COS suivant la topo-} séquence et les modes d'utilisation des terres

Les stocks de COS concernant l'effet interactif du mode d'utilisation des terres et de la topo-séquence sur le stock de carbone organique du sol ont été calculés sur la profondeur $0-30 \mathrm{~cm}$. L'analyse statistique montre que l'effet est significatif au seuil de $5 \%$. Au niveau des formations naturelles, le stock de COS le plus élevé a été obtenu en haut de pente mais pour les plantations, les jachères et les champs, le stock de COS le plus élevé a été obtenu en bas de pente (Figure 3). A tous les niveaux de la topo-séquence, le stock de COS diffère significativement entre les modes d'utilisation des terres. En haut de pente, le stock de COS est significativement plus élevé pour les formations naturelles $(47,83 \mathrm{t} / \mathrm{ha})$ que pour les plantations $(17,87 \mathrm{t} / \mathrm{ha})$, les jachères $(17,89 \mathrm{t} / \mathrm{ha})$ et les champs $(14,94 \mathrm{t} / \mathrm{ha})$. A mipente, les formations naturelles, les plantations, les jachères et les champs ont respectivement stocké $24,52 \mathrm{t} / \mathrm{ha} ; 20,10 \mathrm{t} / \mathrm{ha} ; 14,83 \mathrm{t} / \mathrm{ha}$ et $14,28 \mathrm{t} / \mathrm{ha}$ de carbone organique dans le sol sur $0-30 \mathrm{~cm}$. En bas de pente, les formations naturelles, les plantations, les jachères et les champs ont respectivement stocké $16,16 \mathrm{t} / \mathrm{ha}$; $26,48 \mathrm{t} / \mathrm{ha} ; 23,59 \mathrm{t} / \mathrm{ha}$ et $21,07 \mathrm{t} / \mathrm{ha}$ de carbone organique dans le sol.

\section{Variation du stock de COS en fonction de la texture et des modes d'utilisation des terres}

La Figure 4 met en évidence la variation du stock de COS en fonction de la texture (argile + Limons) et des modes d'utilisation des terres.

Dans le bassin supérieur de Magou, le stock de COS est significativement corrélé avec la teneur en Argile + Limons $\left(\mathrm{R}^{2}>0,5\right)$. Mais la corrélation est plus forte avec un bon ajustement dans les jachères $\left(\mathrm{R}^{2}=0,72\right)$, dans les plantations $\left(\mathrm{R}^{2}=0,70\right)$ et dans les formations naturelles $\left(\mathrm{R}^{2}=0,60\right)$ que dans les champs $\left(R^{2}>0,58\right)$ (Figure 4). 
Tableau 2: Moyennes et écarts-types du stock de COS par mode d'utilisation des terres, par toposéquence et par profondeur.

\begin{tabular}{|c|c|c|c|c|c|}
\hline Modalités & $\begin{array}{c}\text { Moyenne } \\
\text { (t/ha) }\end{array}$ & $\begin{array}{c}\text { Erreur } \\
\text { standard }\end{array}$ & $\begin{array}{c}\text { Dégré de } \\
\text { liberté }\end{array}$ & F observé & p-value \\
\hline \multicolumn{6}{|c|}{ Mode d'utilisation des terres } \\
\hline Formation naturelle & 29,51 a & 6,09 & 3 & 3,34 & 0,04 \\
\hline Plantation & $21,49 \mathrm{ab}$ & 2,99 & & & \\
\hline Jachère & $18,77 \mathrm{ab}$ & 1,88 & & & \\
\hline Champ & $16,76 \mathrm{~b}$ & 2,35 & & & \\
\hline \multicolumn{6}{|l|}{ Topo-séquence } \\
\hline Haut de pente & 24,63 a & 2,79 & 2 & 1,37 & 0,27 \\
\hline Bas de pente & 21,83 a & 1,63 & & & \\
\hline Mi-pente & 18,44 b & 0,88 & & & \\
\hline \multicolumn{6}{|l|}{ Profondeur } \\
\hline $0-10 \mathrm{~cm}$ & $11,63 \mathrm{a}$ & 1,33 & 2 & 31,8 & 0,0001 \\
\hline $10-20 \mathrm{~cm}$ & $5,78 \mathrm{~b}$ & 0,52 & & & \\
\hline $20-30 \mathrm{~cm}$ & $4,23 \mathrm{~b}$ & 0,33 & & & \\
\hline
\end{tabular}

Les chiffres suivis de la même lettre pour chaque facteur ne sont pas significativement différents au seuil de $5 \%$.

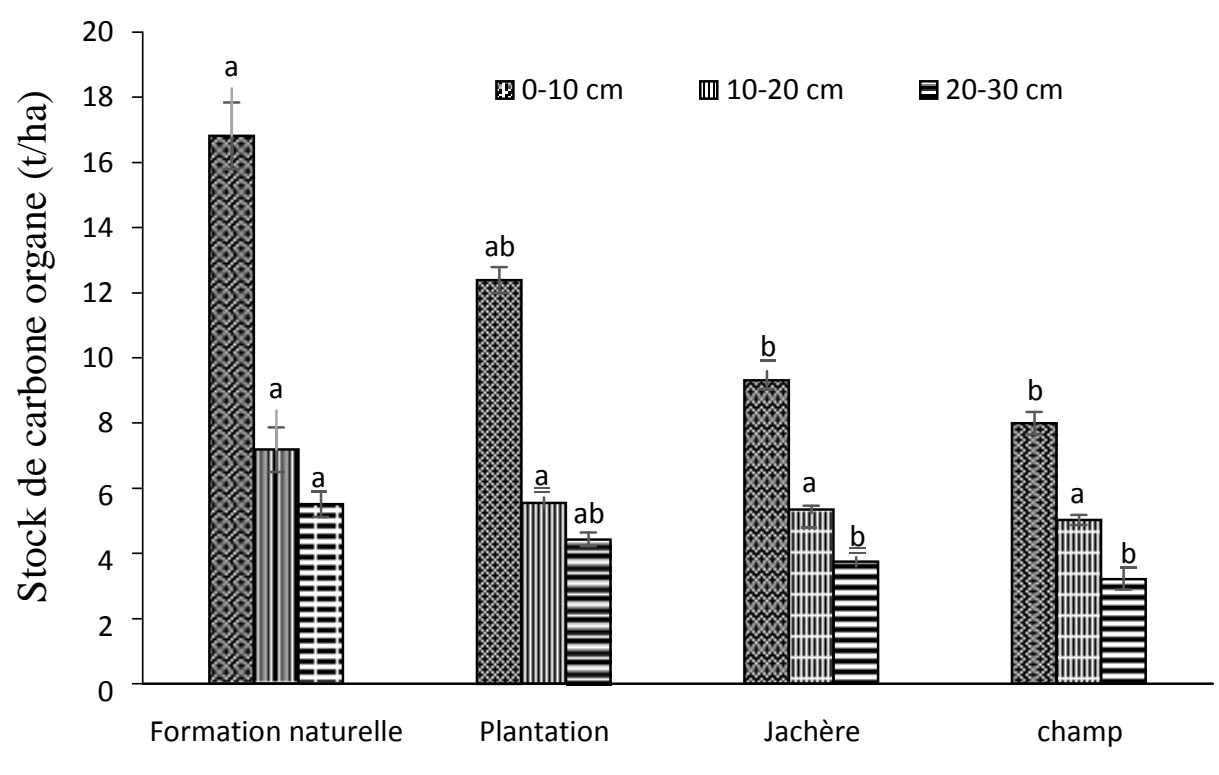

Modes d'utilisation des terres

Figure 2 : Effet combiné du mode d'utilisation et de la profondeur sur le stock de COS.

Pour la même profondeur, les histogrammes portant la même lettre ne sont pas significativement différents au seuil de 5\%. 


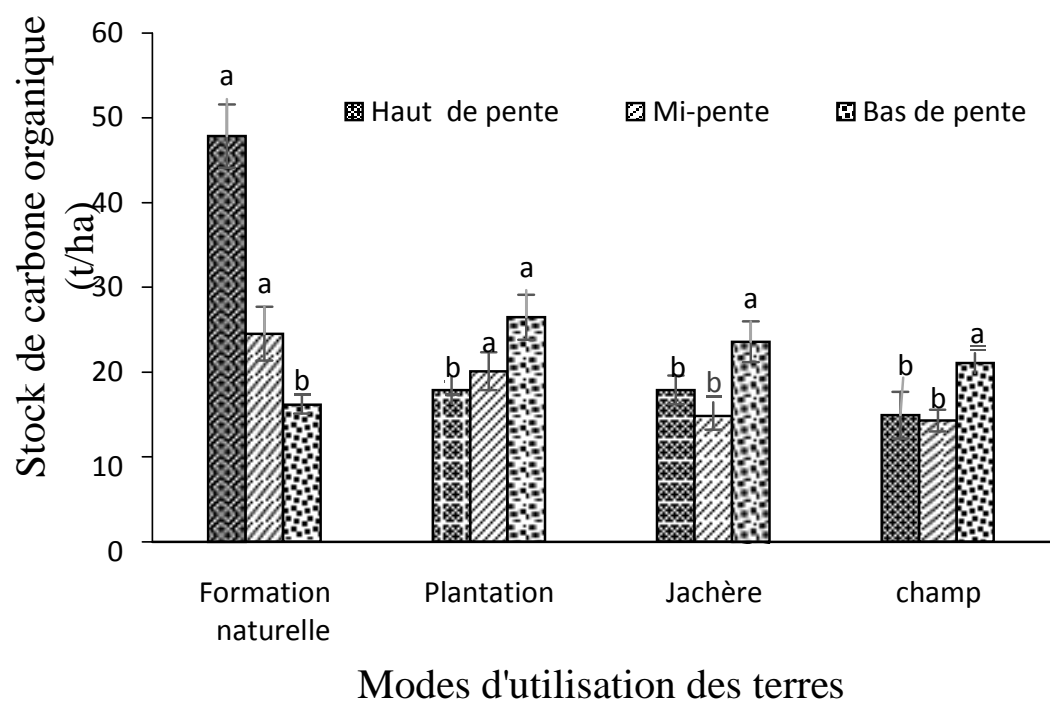

Figure 3 : Effet combiné du mode d'utilisation des terres et de la topo-séquence sur le stock de COS. Pour la même position, les histogrammes portant la même lettre ne sont pas significativement différents au seuil de 5\%.

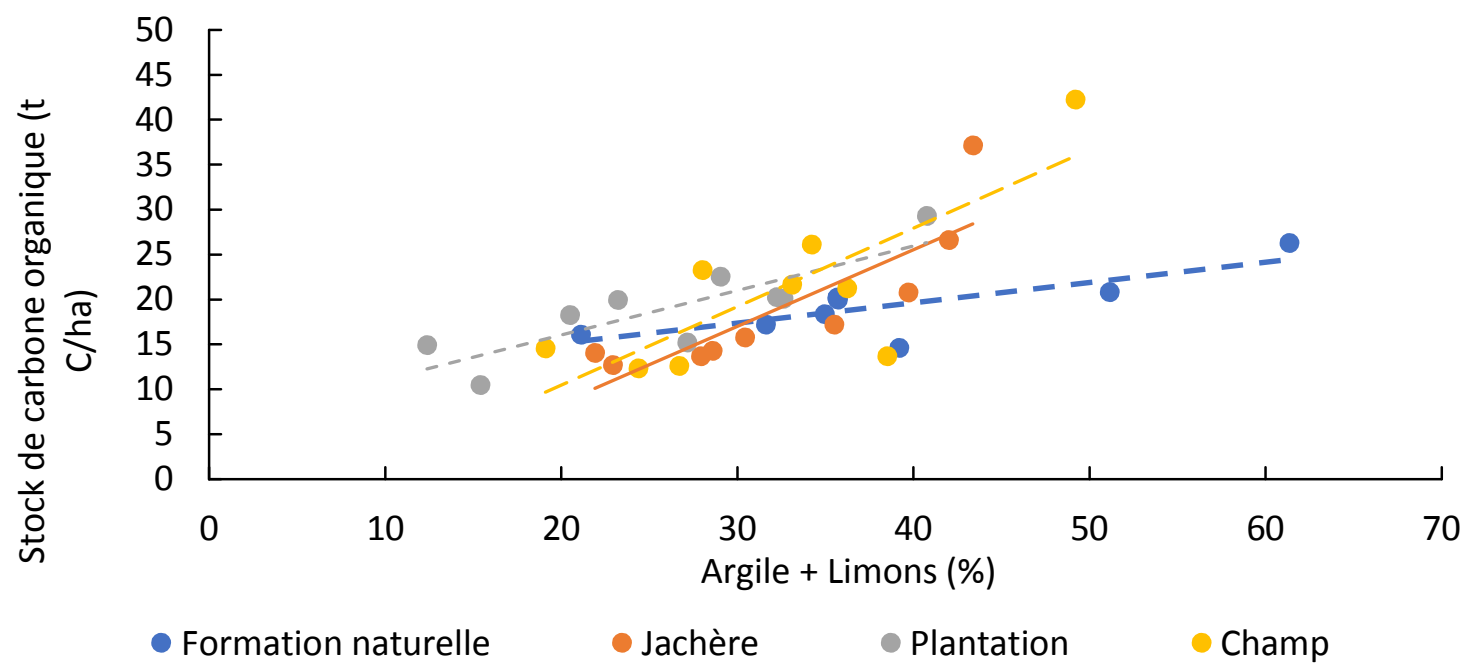

Figure 4: Variation du stock de COS en fonction de la texture (argile + Limons) et des modes d'utilisation des terres.

Tableau 3: Relation entre le stock de carbone organique ( $\mathrm{Sco}$ ) et la proportion d'argile et de limon dans les unités d'occupation.

\begin{tabular}{|c|c|c|}
\hline Mode d'utilisation des terres & Equations & $\mathbf{R}^{2}$ \\
\hline Champ & Sco $(\mathrm{t} \mathrm{C} / \mathrm{ha})=0,8731($ Argile+limons $)-6,9956$ & 0,5897 \\
\hline Jachère & $\operatorname{Sco}(\mathrm{t} \mathrm{C} / \mathrm{ha})=0,8538($ Argile+limon $)-8,6032$ & 0,7238 \\
\hline Plantation & $\operatorname{Sco}(\mathrm{t} \mathrm{C} / \mathrm{ha})=0,4958($ Argile+limon $)+6,1498$ & 0,7007 \\
\hline Formation naturelle & Sco $(\mathrm{t} \mathrm{C} / \mathrm{ha})=0,2265($ Argile+limon $)+10,59$ & 0,6049 \\
\hline
\end{tabular}




\section{DISCUSSION}

\section{Dynamique du stock de COS suivant les modes d'utilisation des terres}

Les résultats montrent que la variation du stock de COS suit un gradient de végétation. En effet, le plus fort stock de carbone organique a été obtenu dans les formations naturelles. Ces résultats s'accordent avec ceux de la FAO (2002) qui a conclu que les écosystèmes forestiers contiennent plus de carbone par unité de surface que tout autre type d'utilisation du sol. La décroissance du stock de COS constatée entre les formations naturelles et les plantations du BSM a été déjà constatée par Marco et al. (2010) en Amérique latine. D'après les travaux de ceux-ci, « la conversion en plantation d'une savane ou d'une forêt naturelle dense provoque une réduction du COS sur les vingt premiers centimètres et à l'inverse, la densification du COS sur la même profondeur ». Les stocks de COS dans les formations naturelles et dans les plantations sont nettement supérieurs à ceux des jachères et champs car les périmètres boisés empêchent les déplacements de sol et d'éléments nutritifs, et emprisonnent le carbone dans le bois et le sol. Outre le fort potentiel de séquestration du carbone des arbres, l'absence ou la rareté des feux de végétation dans ces écosystèmes est un facteur qui favorise le stockage du carbone organique dans le sol. Les activités anthropiques contribuent de façon importante à perturber le cycle naturel du carbone et les équilibres des flux entre les différents réservoirs. En effet, " les changements d'utilisation des terres et l'usage des combustibles fossiles ont été les principales influences de l'homme sur le cycle du carbone $»$ selon Arrouays et al. (2002) et Janzen (2004). C'est pourquoi les stocks de COS des jachères et champs sont plus faibles que ceux des formations naturelles et plantations. $\mathrm{La}$ différence entre les stocks de COS des jachères et ceux des champs indiquerait la mise en place d'un équilibre du statut organique des sols de jachère largement tributaire d'un passé cultural et écologique assez lointain (7 ans), mais beaucoup moins influencé par le passé cultural récent des champs. Le retour d'un sol, qui a subi de nombreux cycles culturaux et qui se situe dans un système de savane, à un équilibre originel semblerait alors extrêmement difficile qui ne pourrait pas être atteint après des jachères de moyenne durée surtout que les techniques de gestion de la fertilité des sols du BSM reposent essentiellement sur les apports minéraux et non organiques. Or, un apport d'engrais et de matière organique a un effet positif sur le rendement quel que soit le type de sol selon Epolyste et al. (2014). Il faut donc tenir compte du système cultural mis en place dans la dynamique du COS. Aholoukpé et al. (2013) l'ont prouvé dans le Sud-Est du Bénin en concluant que « les associations culturales et le recyclage des feuilles d'élagage interviennent dans le stock de matière organique des sols sous palmeraies ».

\section{Dynamique du stock de COS suivant la topo-séquence}

Ahoglè (2018) a démontré que la variation spatiale des stocks de carbone est principalement due aux types de systèmes de culture, à la topographie et la texture des dépôts. Les résultats obtenus dans le BSM confirment le rôle du facteur topographie en ce sens que dans les formations naturelles, le stock de COS augmente avec l'altitude alors que dans les modes d'utilisation des terres plus anthropisés (plantations, jachères et champs), le stock de COS diminue avec l'altitude. Le stockage du COS sur le bassin supérieur de Magou suit donc un gradient hypsométrique dans ces trois derniers modes d'utilisation des terres. Ces résultats corroborent les résultats obtenus par Guo et al. (2006) qui ont montré que «les stocks de carbone diminuaient avec l'altitude et qu'ils étaient fonction de l'inclinaison de la pente, les concentrations les plus élevées se situant en bas de pente ». Dans les sols des écosystèmes anthropisés, le stock le plus important est en bas de pente à la même profondeur car pendant sept ans, les terres ont été mises à nu pour les champs et la recharge organique est devenue très faible avec les activités humaines. L'érosion et le drainage facilitent le transport du COS de l'amont vers l'aval où il s'accumule. Nos résultats confirment ceux de Callesen et al. (2003) qui ont mis en évidence que le drainage est un 
facteur important à considérer puisqu'il influence la capacité de séquestration du carbone avec les stocks les plus importants dans les sites les moins bien drainés. Il faut donc du temps, même avec le repos des terres pour le rétablissement de l'équilibre.

Dans les formations naturelles, les fortes concentrations de COS en haut de pente sont liées à l'âge de ces formations végétales, à l'inexploitation et au faible drainage. En effet, sur deux des trois sites (Ouankou et Pingou), la rugosité de la surface du sol due au fort taux d'affleurement rocheux en haut de pente rend difficile le travail de la terre et le déboisement puis réduit le drainage. De même, ces hauts de pente très proches des habitations reçoivent quotidiennement les matières fécales de toute nature.

La morphologie des sols est un facteur non négligeable dans la dynamique du COS. En haut de pente, le sol, à texture limonosableuse, est vite lessivé et est très peu humide pour faciliter la minéralisation de la matière organique. La stabilité structurale du sol est plus importante pour résister à l'érosion et au transport du carbone vers l'aval. Kombiénou et al. (2015) ont obtenu des résultats similaires en constatant que dans le Nord-Ouest du Bénin, les teneurs en matière organique des sols des plateaux sont plus faibles que celles des sols des versants des montagnes. Cela est lié à la faible exploitation de ces versants. En se référant aux travaux d'Amoussou et al. (2015) qui ont prouvé une baisse de l'écoulement dans le BSM, on peut comprendre dans une certaine mesure le mauvais drainage qui serait à l'origine des stocks élevés de COS en haut de pente.

\section{Dynamique du stock de COS suivant la profondeur}

Dans le bassin supérieur de Magou, le stock de carbone organique décroît de la surface à la profondeur. En effet, la plus forte et la plus faible moyennes de stock de COS ont été respectivement obtenues sur la profondeur $0-10 \mathrm{~cm}(11,63 \mathrm{t} / \mathrm{ha})$ et la profondeur $20-30 \mathrm{~cm}$ $(4,23 \mathrm{t} / \mathrm{ha})$. La diminution du stock de COS de la surface vers la profondeur dans le BSM est en accord avec les résultats d'Aholoukpè et al.,
(2016) qui ont conclu que verticalement, les stocks de carbone des sols présentent un gradient de la surface vers la profondeur et le gradient peut parfois être très accusé, en particulier en sol forestier. De même, une étude réalisée dans l'écosystème des iliçaies du Moyen Atlas marocain par Boulmane et al. (2014) montre que « les stocks de COS diminuent avec la profondeur en suivant une courbe exponentielle à exposant négatif ». L'importance du carbone organique dans les premières dizaines de centimètres est liée à la teneur en matière organique accumulée à ce niveau par la litière, la biomasse racinaire et la stabilité des colloïdes organiques contrairement aux colloïdes minéraux (argiles).

\section{Dynamique du stock de COS suivant la texture}

D'après les résultats, il y a une relation entre la teneur en éléments fins et la teneur en carbone des sols. L'augmentation de la proportion en éléments fins favorise un accroissement des teneurs en matières organiques. Dans les formations naturelles et dans les plantations, la teneur en carbone est positivement corrélée avec la teneur en argile. Mais cette corrélation n'est pas significative dans les plantations. Cette relation entre la texture et la teneur en carbone est à rapprocher des travaux réalisés par Alohoutadé (2018). En effet, dans une étude sur un bassin versant agricole sur le plateau Adja au Bénin, ce dernier a établi une corrélation entre teneur en carbone et teneur en éléments fins $(<20 \mu \mathrm{m})$. Ces résultats sont à nuancer car les sols travaillés échappent à cette loi. En effet, nous avons obtenu dans les champs et dans les jachères une corrélation négative entre la teneur en argile et le taux de carbone. Cela suppose que dans ces écosystèmes, un sol à fort taux d'argile est pauvre en carbone. Dans les champs, ce constat peut s'expliquer par deux faits : i) le retournement continuel du sol sur en profondeur de $30 \mathrm{~cm}$ environ a fait remonter à la surface l'horizon d'accumulation qui est beaucoup plus riche en argile; ii) sur le bassin supérieur de Magou les pratiques agricoles adoptées (surtout le brûlis de la végétation 
morte ou vivante en début de saison de culture) appauvrissent fortement le pool organique du sol. Par ailleurs, le constat dans les jachères peut être dû au fait qu'après une longue période de mise culture, la courte période de jachère n'est pas encore suffisante pour impacter significativement le stock organique du sol. Selon Kouelo (2016), il faut au moins 30 ans de jachère pour combler les impacts négatifs de 10 ans d'utilisation du sol en Afrique subsaharienne. Aussi, les jachères sont-elles affectées par des feux de végétation.

\section{Conclusion}

A l'issue de cette étude qui visait à évaluer l'impact des modes d'utilisation des terres sur le stock de COS dans le bassin supérieur de Magou, il ressort que le mode d'utilisation des terres, la profondeur, la toposéquence et la texture influencent significativement les stocks de COS dans ledit bassin. Le stock de COS suit un gradient de végétation avec les plus forts taux dans les formations naturelles et les plus faibles dans les champs en passant respectivement par les plantations et les jachères. Verticalement, le stock de COS diminue de la surface vers la profondeur. Par rapport à la topo-séquence, le stock le plus élevé est globalement en haut de pente alors que le plus faible est à mi- pente. Toutefois, dans les modes d'utilisation les plus anthropisés, la tendance est renversée au point où le stock diminue du bas de pente vers le haut de pente. Au plan textural, le stock de COS est positivement corrélé avec la teneur en éléments fins. Au regard des impacts possibles de la dynamique du COS sur la fertilité des sols et sur le cycle du carbone, des mesures correctives s'imposent non seulement pour une gestion durable des terres dans le bassin supérieur de Magou mais aussi pour une gestion plus efficiente des problèmes environnementaux.

\section{CONFLIT D'INTERETS}

Les auteurs déclarent qu'il n'y a aucun conflit d'intérêts à signaler dans le cadre de ce travail.

\section{CONTRIBUTIONS DES AUTEURS}

CCA a élaboré le protocole de recherche, conduit les travaux de terrain, interprété et discuté les résultats avant de procéder à la rédaction. AGZ a participé aux travaux de terrain, contribué à l'analyse des échantillons de sol et à la lecture. TMA s'est occupé de l'analyse des échantillons et du traitement statistique. AHA a contribué à la définition de la technique d'échantillonnage et de prélèvement puis à la lecture. $\mathrm{ABT}$ a contribué à la correction de la méthodologie et à la lecture de l'article. JGD a contribué à la lecture de l'article.

\section{REMERCIEMENTS}

Les auteurs remercient tous les agriculteurs, les guides et les chefs de village qui ont accepté participer à cette étude sans oublier les techniciens qui ont aidé à la collecte des échantillons et aux analyses physicochimiques au Laboratoire.

\section{REFERENCES}

Ahoglè AMA. 2018. Effet des systèmes de culture et de la pente sur le statut organique du sol sur bassin versant de Kiti (commune de Djidja au Bénin). Mémoire de Master professionnel, Faculté des Sciences Agronomiques/Université d'AbomeyCalavi, 76p.

Aholoukpè HNS, Amadji GL, Blavet D, Chotte JL, Deleporte P, Dubos B, Flori A, Jourdan C. 2016. Effet de la gestion des feuilles d'élagage du palmier à huile sur le stock de carbone et les propriétés physicochimiques du sol dans les palmeraies villageoises du Bénin. Biotechnologie Agronomie Société et Environnement, 20(2) : 171-182.

Aholoukpé H, Vissoh VP, Amadji G, Deleporte P, Dubos B, Nodichao L, Balavet D. 2013. Typologie des plantations villageoises de palmier à huile (Elaeis guineensis Jacq.) dans le département du Plateau au Bénin. International Journal of 
Biological and Chemical Sciences, 7(3) : 978-999.

Akplo TM, Kouelo AF, Houngnandan P, Benmansour M, Rabesiranana N, Mabit L, Ahoglè Agassin MA, Alohoutade FM. 2017. Effect of tillage and mulching on soil water erosion in linsinlin watershed, centre of benin. Journal of Experimental Biology and Agricultural Sciences, 5(4): 515-524.

DOI: http://dx.doi.org/10.18006/2017.5

(4).515.524

Alohoutadé MF. 2018. Effet des systèmes de culture et de la pente sur la dégradation du sol du bassin versant de Kpacomey (commune d'Aplahoué au Bénin). Mémoire de Master professionnel, Faculté des Sciences Agronomiques/Université d'AbomeyCalavi, 60p.

Amoussou E, Totin HSV, Vissin EW, Mahe G, Houndénou C, Boko M. 2015. Modélisation pluie-débit pour la gestion intégrée des ressources en eau (GIRE) de surface dans le bassin-versant de la Volta au Bénin. Revue de géographie du laboratoire Leïdi, 13: 282-296

Arrouays D, Balesdent J, Germon JP, Jayet PA, Soussana JF, Stengel P. 2002. Stocker du carbone dans les sols agricoles de France ? Expertise scientifique collective. Institut national de la recherche agronomique (INRA). France. 322 p.

Balesdent J, Chenu C, Balabane M. 2000. Relationship of soil organic matter dynamics to physical protection and tillage. Soil and Tillage Research, 53: 215-230. https://doi.org/10.1016/S01671987(99)00107-5

Bello OD, Saïdou A, Ahoton EL, Avaligbé JFY, Ezin AV, Akponikpè PBI, Aho N. 2017. Assessment of organic carbon stock in cashew plantations (Anacardium occidentale L.) in Benin (West Africa). International Journal of Agriculture and Environmental Research, 3(4): 34713495.

Boulmane M, Santa-Regina I, Khia A, Oubrahim H. 2014. Éstimation du stock de carbone organique dans l'écosystème des iliçaies du Moyen Atlas marocain. Nature and Technology, (11): 6.

DOI

https://www.asjp.cerist.dz/en/downArticl e/47/8/11/40768

Callesen L, Liski L, Raulund-Rasmussen K, Olsson MT, Tau-Strand L, Vesterdal L, Westman CL. 2003. Soil carbon stores in Nordic weil drained forest soils relationships with climate and texture class ». Global Change Biology, 9: 358370. https://doi.org/10.1046/j.13652486.2003.00587.x

Callesen L, Liski L, Raulund-Rasmussen K., Olsson MT, Tau-Strand L, Vesterdal L, Westman CL. 2003. «Soil carbon stores in Nordic weil drained forest soils relationships with climate and texture class ». Global Change Biology, 9 : 358370.

Dagnelie P. 1998. Statistique théorique et appliquée vol. 2. Paris, De Boeck \& Larcier, Belgique, 659p.

DSA (Direction des statistiques agricoles), 2017. Evolution de la production agricole. Cotonou, $37 \mathrm{p}$.

Epolyste A, Yacouba H, Zombre P N. 2014. Etat de fertilité des sols du périmètre irrigué gravitaire de Gouran dans la vallée du Sourou au Burina Faso : Constats et perspectives. International Journal of Biological and Chemical Sciences, 8(5), 2119-2131.

FAO. 2002. Rapport sur les ressources en sols du monde. «La séquestration du carbone dans le sol pour une meilleure gestion des terres ». Rome, 70p.

Fermont AM, van Asten PJA, Giller KE. 2008. Increasing land pressure in East Africa: the changing role of cassava and consequences for sustainability of farming systems. Agric. Ecosystem. Environment, 128 : 239-250.

Glèlè Kakaï R, Salako VK, Lykke AM. 2016. Techniques d'échantillonnage en étude de végétation. Annales des Sciences Agronomiques 20 - spécial Projet Undesert-UE: 1-13 ISSN 1659-5009.

Guo G, Zhang H, Araya K, Jia H, Ohomiya K, Matsuda J. 2006. Improvement of 
saltaffected soils, Part 3: Specifie Heat of Salt-affected soils. Biosystems Engineering, $\quad$ 97:413-418. https://doi.org/10.1016/j.biosystemseng. 2006.11.007

Herfurth D. 2015. Impact des pratiques de gestion sur le stockage du Carbone dans le sol des écosystèmes prairiaux. Thèse unique de doctorat, Université d'Auvergne, France, 203p.

Janzen HH. 2004. Carbon cycling in earth Systems - a soil science perspective. Agricultuer, Ecosystems and Environment, 104: 399-417.

Kombienou PD, Arouna O, Azontondé AH, Mensah G A, Sinsin BA. 2015. Caractérisation du niveau de fertilité des sols de la chaîne de l'Atakora au NordOuest du Bénin. Journal of Animal \&Plant Sciences, 25(2) : 3836-3856.

Kombienou PD, Arouna O, Azontonde HA, Mensah GA, Sinsin AB. 2014. Influences des activités agricoles sur la fertilité des sols de la chaîne de l'Atakora au nordouest du Bénin. Rev. Sc. Env. Univ., Lomé (Togo), 11 :1812-1403., LaRBE, 1:381-404.

Kouelo AF, 2016. Effets des pratiques culturales sur la dégradation du sol au niveau des trois bassins versants du sud Bénin. Thèse de Doctorat en Sciences Agronomiques, Universités d'AbomeyCalavi, Bénin, 239 pages.

Kouelo AF, Houngnandan P, Azontondé HA, Benmansour M, Rabesiranana N, Mabt L. 2015. Assessment of the level of soil degradation in three watersheds affected by intensive farming practices in Benin. Journal of Experimental Biology and Agricultural Sciences, 3(6): 529-540.

Marco P, Henry M, Blavet D, Bernoux M. 2010. Variation des stocks de carbone organique du sol après plantation forestière : essai de méta-analyse incluant quelques situations d'Amérique latine. Bois et Forêts des Tropiques, 305: 21-32.
Nukpo A. 2016. Fondamentaux de Géographie du Bénin, ÉPA/CÉRADE, Porto-Novo,

$69 \mathrm{p}$.

Saïdou A, Balogoun I, Koné B, Gnanglè CP, Aho N. 2012. Effet d'un système agroforestier à karité (Vitellaria paradoxa c.f. gaertn) sur le sol et le potentiel de production du maïs (Zea mays L.) en zone Soudanienne du Bénin. International Journal of Biological and Chemical Sciences, $\quad$ 6(5): $\quad$ 2066-2082. DOI:http://dx.doi.org/10.4314/ijbcs.v6i5. 16

Saïdou A, Kossou D, Azontondé A, Hougni DGJM. 2009. Effet de la nature de la jachère sur la colonisation de la culture subséquente par les champignons endomycorhiziens : cas du système 'jachère' manioc sur sols ferrugineux tropicaux du Bénin, International Journal of Biological and. Chemical Sciences, 3(3): 587-589.

Tekalign T, Haque I, Aduayi EA. 1991. Soil, plant, water, fertilizer, animal manure and compost analysis manual. Plant Science Division Working Document 13, ILCA, Addis Ababa, Ethiopia.

Tosser V, Eglin T, Bardy M, Besson A, Martin M. 2013. Évaluation des stocks de carbone organique des sols cultivés de France. Etude et Gestion des Sols, 21: 723.

Walkley A, Black CA. 1934. An examination of the Degtjareff method for determining soil organic matter and a proposal modification of the chromic acid titration method. Soil Science, 37 : 29-38. 